\title{
SPIRAL MICROFLUIDICS DEVICE FOR CONTINUOUS FLOW PCR
}

\author{
Reza Salemmilani \\ Microfluidics \& Lab-on-a-chip Research Group \\ Mechanical Engineering Department \\ Ihsan Dogramaci Bilkent University \\ Ankara 06800 TURKEY \\ Email: reza.salemmilani@bilkent.edu.tr
}

\author{
Barbaros Cetin* \\ Microfluidics \& Lab-on-a-chip Research Group \\ Mechanical Engineering Department \\ Ihsan Dogramaci Bilkent University \\ Ankara 06800 TURKEY \\ Email: barbaros.cetin@bilkent.edu.tr
}

\begin{abstract}
Polymerase-chain-Reaction $(P C R)$ is a thermal cycling (repeated heating and cooling of PCR solution) process for DNA amplification. PCR is the key ingredient in many biomedical applications. One key feature for the success of the PCR is to control the temperature of the solution precisely at the desired temperature levels required for the PCR in a cyclic manner. Microfluidics offers a great advantage over conventional techniques since minute amounts of PCR solution can be heated and cooled with a high rate in a controlled manner. In this study, a microfluidic platform has been proposed for continuous-flow PCR. The microfluidic device consists of a spiral channel on a glass wafer with integrated chromium microheaters. Sub-micron thick microheaters are deposited beneath the micro-channels to facilitate localized heating. The microfluidic device is modeled using COMSOL Multiphysics $囚$. The fabrication procedure of the device is also discussed and future research directions are addressed. With its compact design, the proposed system can easily be coupled with an integrated microfluidic device to be used in biomedical applications.
\end{abstract}

\section{NOMENCLATURE}

$c_{p} \quad$ specific heat

$k$ thermal conductivity

u velocity vector

$P$ pressure

$r$ geometric design parameter shown in Fig. 1

*Address all correspondence to this author.

$T$ temperature

$T_{\text {ave }}$ average temperature

$\mu$ viscosity

$\rho$ density

$\phi \quad$ geometric design parameter shown in Fig. 1

\section{INTRODUCTION}

There are many methods for detecting pathogens such as whole cell detection, antibody detection and DNA/RNA detection. DNA assays, owing to their sensitivity and specificity have a clear advantage over the two other methods [1]. In DNA assays, DNA content of the sample is first extracted and then amplified. Amplification can be performed using several methods, the most popular of which is Polymerase-Chain-Reaction (PCR). PCR is a reaction in which specific fragments of DNA are copied exponentially in a thermal cycling process [2]. Different protocols for PCR have been proposed. Real-time PCR is a technique, using which specific targeted types of DNA are copied, detected and quantified simultaneously [3]. The process involves heating the sample containing DNA and the PCR solution (Polymerase enzyme, primers, buffer etc.) to $90-96^{\circ} \mathrm{C}$ for $20-30 \mathrm{sec}-$ onds to melt the DNA and separate its strands, then cooling it to $50-65^{\circ} \mathrm{C}$ in order to allow binding of the primers to the specific strand which is to be copied, followed by heating to around $72{ }^{\circ} \mathrm{C}$ for replicating the primed strand to double-strands followed by elongation of the newly copied DNA [4]. This process is cyclic and is repeated generally 20-40 times based on the number of amplified strands required for detection. 
There are two different approaches in designing microfluidic platforms for PCR. One is having a stationary reaction chamber, temperature of which is varied according to the PCR protocol. The other approach is a continuous flow system in which three distinct temperature zones are generated on the chip and the flow moves through these zones in cycles. One main advantage of the continuous flow system over its stationary counterpart is the reduced thermal inertia of the system, as only the fluid needs to be heated and cooled not the whole chamber. This reduced inertia results in faster temperature cycling which enhances the overall performance of the device [5].

Several different microchannel architectures have been investigated by researchers. The early continuous flow PCR chips used a serpentine channel passing through three different temperature regions. An important drawback of this design is the order of heating zones which forces a single-strand DNA emerging from the melting zone to pass through the elongation stage before it can enter the annealing zone for binding with primers. This unwanted stay at elongation temperature takes place in every other cycle, resulting in formation of double-strand DNA and thereby decreased PCR efficiency [6]. Some groups have reported spiralchannel configuration for the PCR chip. This configuration has the potential to effectively eliminate double-strand DNA formation due to the correct order of heating stages at every cycle. Zhang et al. [5] reported a continuous flow polymerase chain reaction chip by embedding channels inside copper heating blocks. Another group [7] designed and fabricated a PDMS-based spiral channel PCR chip with integrated platinum heaters and a fan for heating and cooling.

As for the heating and cooling of the chip, many groups $[8,9]$ have used heating blocks and peltier units. However, large thermal inertia of these units prevents fast response of the system and increases the overall size of the device. Integrated micro heaters have a much smaller thermal inertia and are extremely small in size and hence appear to be more convenient for microchip applications. However, one drawback is the difficult and expensive methods involved in their fabrication. Some groups have used titanium and Nichrome thin films as heating elements for PCR reactors [5].

In this study a spiral-channel microfluidics device for realtime PCR is analysed. The device consists of $100 \mu \mathrm{m}$ wide and deep microchannels inside a glass substrate with $400 \mathrm{~nm}$ thin film chromium heaters beneath the channels to provide localized heating in order to eliminate the need for forced cooling. The flow and temperature fields are simulated within the device by using COMSOL Multiphysics $囚$. The average temperature within the microchannel is determined and several design parameters are optimized for enhanced performance. The fabrication procedure of the proposed design is also discussed and future research directions are addressed.

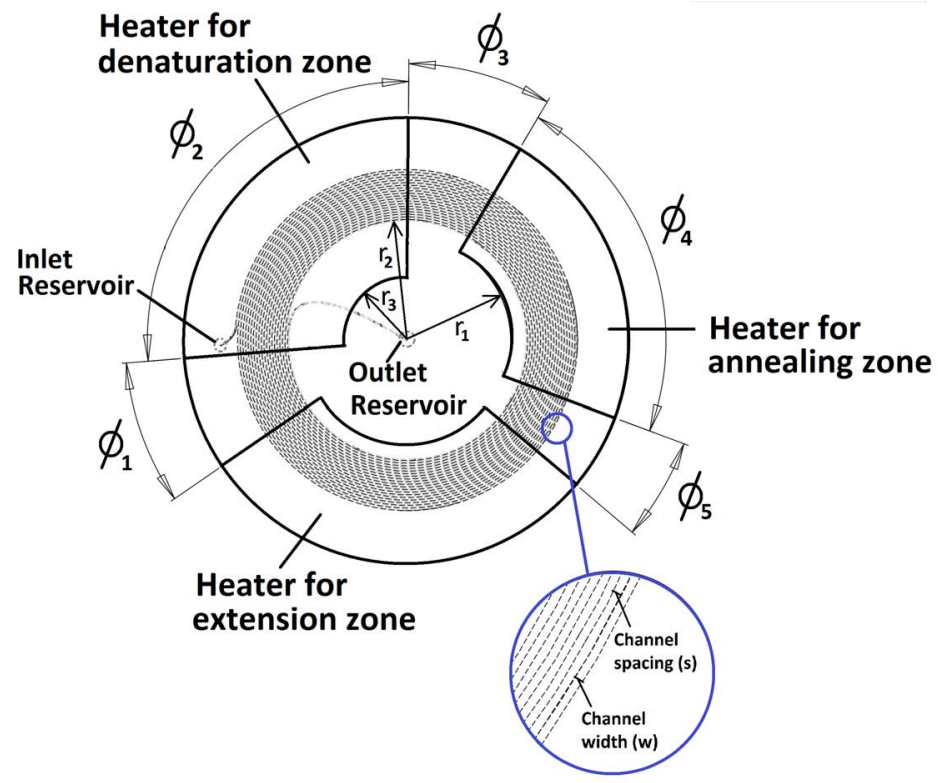

Figure 1. Schematic Drawing of the Device

Table 1. Geometric Parameters for Different Cases

\begin{tabular}{cccccc}
\hline Design Par. & Case 1 & Case 2 & Case 3 & Case 4 & Case 5 \\
\hline$\phi_{1}$ & $15^{\circ}$ & $15^{\circ}$ & $30^{\circ}$ & $30^{\circ}$ & $30^{\circ}$ \\
$\phi_{2}$ & $105^{\circ}$ & $105^{\circ}$ & $95^{\circ}$ & $95^{\circ}$ & $95^{\circ *}$ \\
$\phi_{3}$ & $20^{\circ}$ & $20^{\circ}$ & $30^{\circ}$ & $30^{\circ}$ & $30^{\circ}$ \\
$\phi_{4}$ & $105^{\circ}$ & $105^{\circ}$ & $80^{\circ}$ & $80^{\circ}$ & $80^{\circ * *}$ \\
$\phi_{5}$ & $10^{\circ}$ & $10^{\circ}$ & $20^{\circ}$ & $20^{\circ}$ & $20^{\circ}$ \\
$r_{1}$ & $8.8 \mathrm{~mm}$ & $7.0 \mathrm{~mm}$ & $7.0 \mathrm{~mm}$ & $7.0 \mathrm{~mm}$ & $7.0 \mathrm{~mm}$ \\
$r_{3}$ & $8.8 \mathrm{~mm}$ & $7.0 \mathrm{~mm}$ & $7.0 \mathrm{~mm}$ & $5.1 \mathrm{~mm}$ & $5.1 \mathrm{~mm}$ \\
\hline
\end{tabular}

* Denaturation heater is split into three subzones: $25^{\circ}+45^{\circ}+25^{\circ}$

** Annealing zone is split into two subzones: $40^{\circ}+40^{\circ}$

*** Extension zone is split into two subzones: $65^{\circ}+40^{\circ}$

\section{ANALYSIS}

For the scope of our simulation, PCR is a thermal cycling of a certain buffer solution. The thermal steps for a typical PCR protocol is [10]:

(1) Denaturation step: $90-96^{\circ} \mathrm{C}$ for $20-30$ seconds

(2) Annealing step: $50-65^{\circ} \mathrm{C}$ for $20-30$ seconds

(3) Extension step: $72^{\circ} \mathrm{C}$ for $20-30$ seconds

To ensure the proper thermal cycling, a device composed of a spiral microchannel network is studied as proposed in the lit- 
erature $[5,7]$, and several design parameters are optimized for enhanced performance. The schematic drawing of the system is given in Fig. 1. The spiral channel starts from an outer radius and moves towards the center of the wafer as it travels across different temperature zones in a cyclic manner. To ensure three different temperature zones, three different heater sections are created each of which has a different length in $\phi-$ and $r$-directions. Five different cases are considered each of which is chosen for a better performance. In the fifth case, seven heating sections are used instead of three to study the effect of increasing the number of heaters on the overall performance of the device. Different geometric parameters considered for all cases are given in Tab. 1 . For all cases, $r_{2}$ is taken as $8.6 \mathrm{~mm}$.

The flow field within the microchannels is governed by the incompressible form of continuity and NavierStokes equations:

$$
\begin{gathered}
\nabla \cdot \mathbf{u}=0 \\
\rho \mathbf{u} \cdot \nabla \mathbf{u}=-\nabla P+\mu \nabla^{2} \mathbf{u}
\end{gathered}
$$

subjected to the no-slip boundary condition at the channel walls, uniform velocity at the inlet and zero pressure boundary condition at the exit. The inlet velocity is determined such that it ensures the required time intervals for each step based on the average velocity within the microchannel.

The temperature within the microchannels is governed by the energy equation as follows:

$$
\rho c_{p} \mathbf{u} \cdot \nabla T=k \nabla^{2} T
$$

The heat conduction within the fused silica wafer and PDMS cover is governed by the conduction equation as follows:

$$
k_{i} \nabla^{2} T=0
$$

where $k_{i}$ is the thermal conductivity of the domain of interest. In a real application, the heat will be generated within the heater section as a result of the resistive heating of the electrode structure in the form of a volumetric heat generation. However, in our computational model, the resistive heating of the electrodes is not modeled, and isoflux boundary condition is given on the electrode surface. The required flux values which generate the desired temperature zones are determined by an iterative procedure. For the top, bottom and side surfaces of the device convective boundary condition is used. A lower value is assigned for the bottom surface since the device will be on a platform and not subjected to air movement. At the inlet of the microchannels, specified temperature of $90^{\circ} \mathrm{C}$ is assigned and for the exit of the microchannel, convective outflow boundary condition is designated.

Once the temperature field is determined, the average temperature within the microchannel can be obtained as follows:

$$
T_{\text {ave }}=\frac{1}{A} \int_{A} T d A
$$

Moreover, dividing the average temperature by the average velocity gives the average temperature within the microchannel as a function of time.

\section{NUMERICAL MODELING}

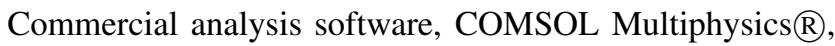
which is based on finite element method, is used to determine the flow and temperature fields. The density and the viscosity of water also depend on temperature. So, the flow field and temperature field are coupled. However to ensure convergence, two flow fields are solved in an iterative manner. First, the flow field is solved based on the initial zero velocity and room temperature. After that, the energy equation is computed with the known velocity values. Then, the flow field and temperature fields are re-computed in an iterative manner until the converged solution is obtained. The simulations were carried out on a SuperServer Workstation (Intel Xeon X5687, Quad core, 3.60GHz, 96GB RAM). Finer meshes are generated for the microchannel structure and a relatively course mesh is chosen for the solid regions. Approximately 1 million elements are used for each case. Typical run-time for one case (after determining the required heat flux values for each zone) is about 20 mins. The justification for the computed results is based on the inspection of the velocity and temperature profiles within the microchannels. Since the flow is laminar, parabola-like velocity profiles are expected and observed as a result of the computations.

\section{RESULTS AND DISCUSSION}

The flow and temperature fields are simulated with the aforementioned boundary conditions. For the transport properties of the PCR solution, those of water which is defined in the COMSOL Materials library as a function of temperature are used. The thermal conductivity of the fused silica is defined using the material library, and thermal conductivity of the PDMS is taken as $0.2 \mathrm{~W} / \mathrm{mK}$.

In Case 1, the geometry with the parameters given in Tab. (1) is investigated. Fig. (2) shows the average temperature of the PCR sample as a function of time as it flows through the channels. It is observed that the desired constant temperature behavior 


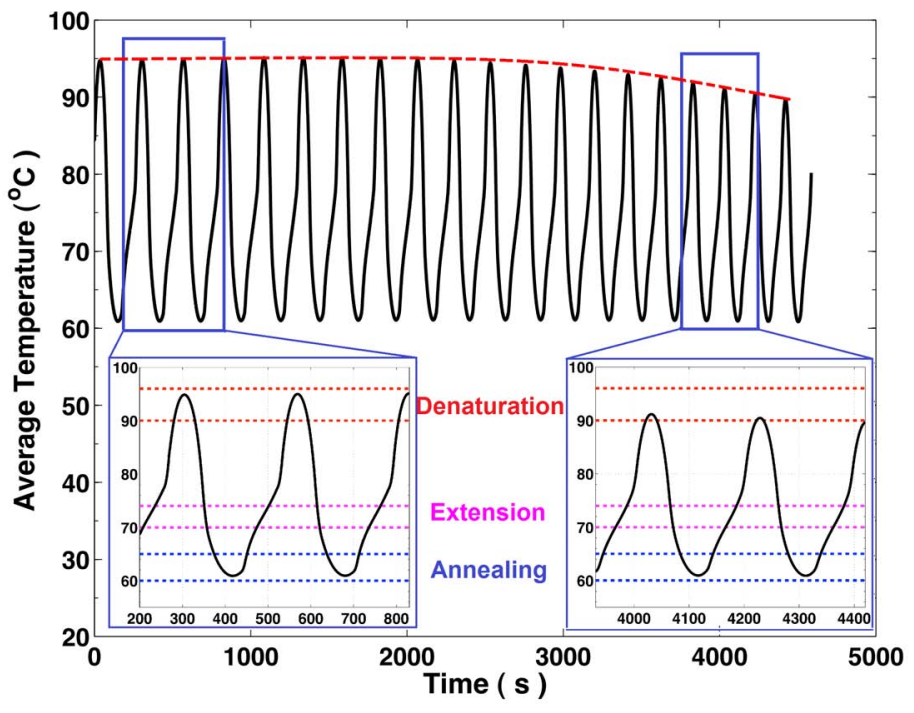

Figure 2. Average Temp. Within the Microchannel (CASE 1)

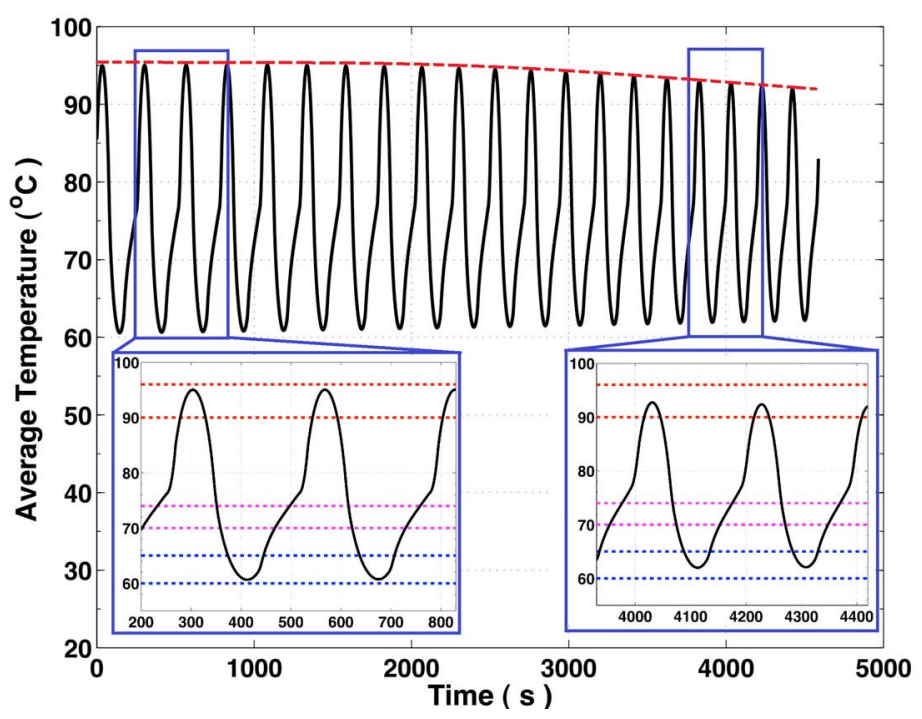

Figure 3. Average Temp. Within the Microchannel (CASE 2)

within the zones is not satisfied. Moreover, in the final few cycles, the mean temperature in the denaturation zone drops significantly (for the clarity of this trend a red dashed line is included in the figure). To reduce the undesired radial temperature gradient observed in Fig. (2), the heaters are further extended towards the center of the chip by reducing $r_{1}$ and $r_{3}$ as denoted in Tab. (1) for Case 2. Fig. (3) shows the average temperature as function of time for this case. Comparing this Case 2 with Case 1 (observing the dashed red lines), it is obvious that the radial gradient in the denaturation zone is reduced. However, the problem of not

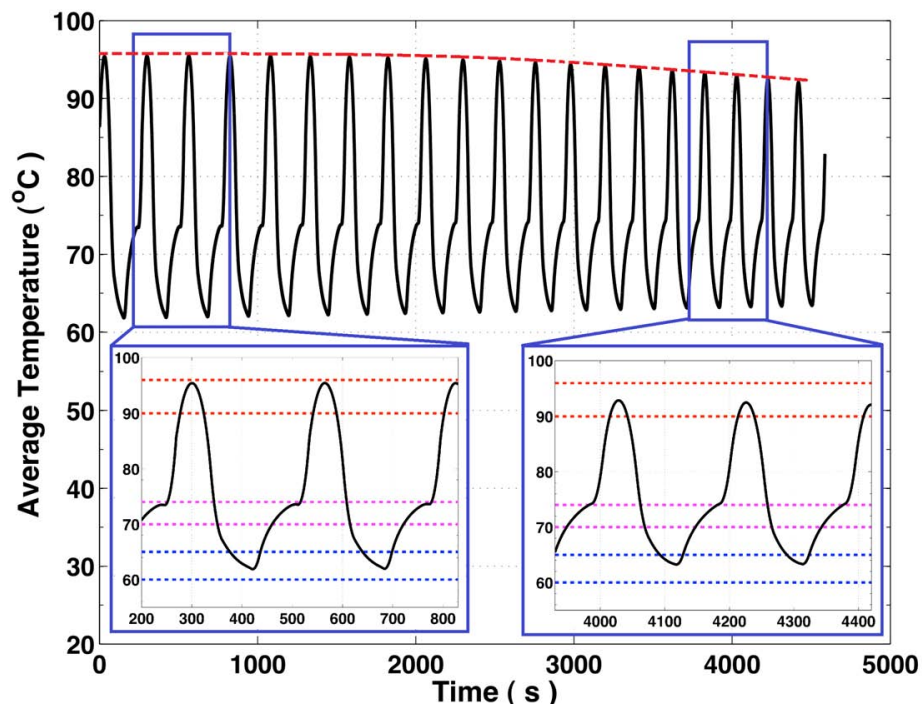

Figure 4. Average Temp. Within the Microchannel (CASE 3)

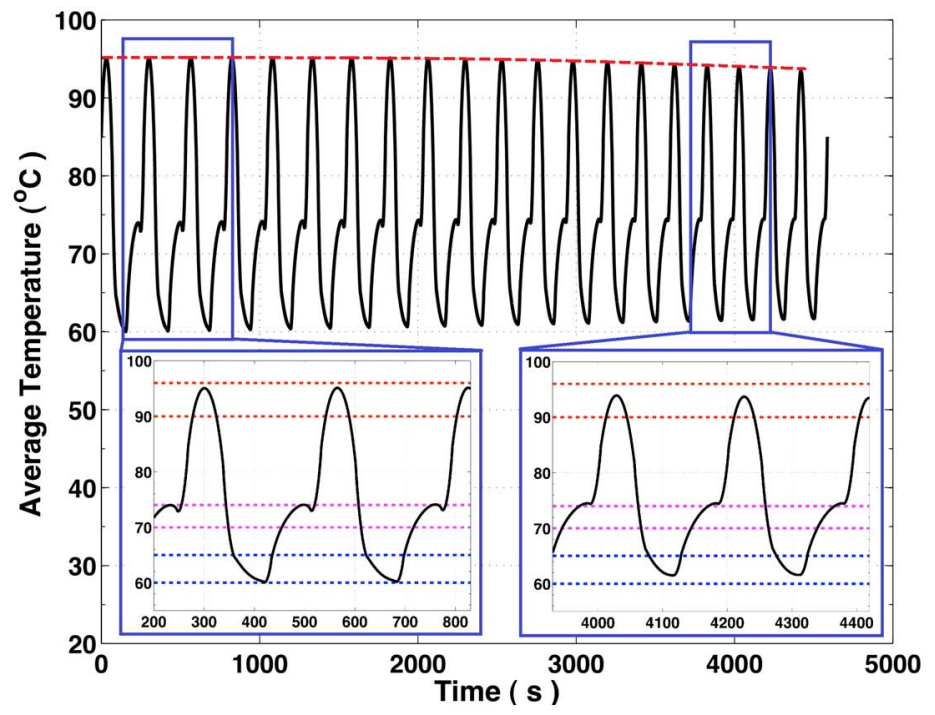

Figure 5. Average Temp. Within the Microchannel (CASE 4)

having a reasonably non-varying temperature in the zones still remains. The main reason for this problem is the thermal interference from the neighboring zones. To solve this problem, in Case 3, the size of the heaters and their angular spacing with respect to each other is modified. Fig. (4) demonstrates the reduced variation in the temperature in each of the three zones. Better thermal performance may be obtained by opening 300 micron trenches within the transition zones between the heaters. Case 4 exploits this possibility and the results are shown in Fig. (5). The trenches reduce the conduction heat transfer area between 


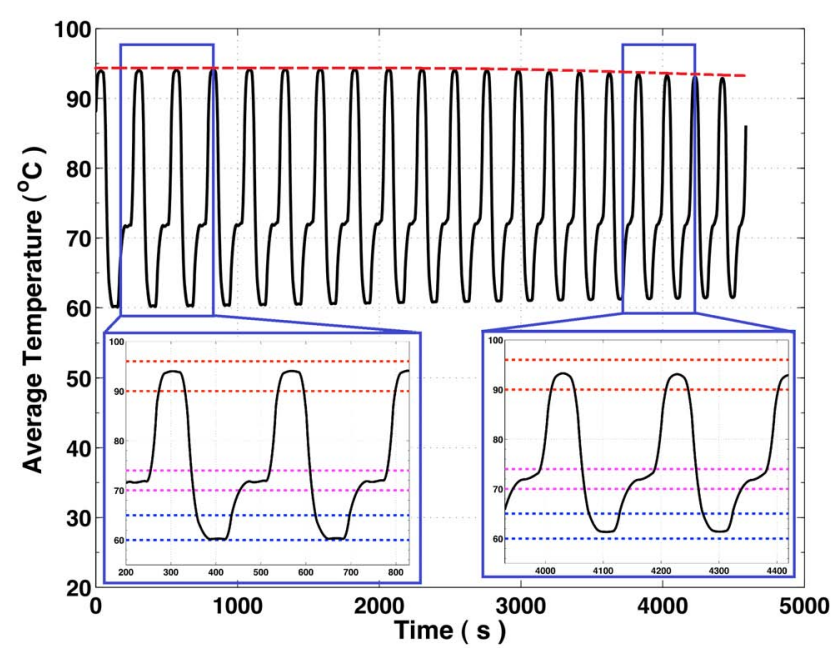

Figure 6. Average Temp. Within the Microchannel (CASE 5)

the zones and reduce the thermal footprint of the neighboring zones. Also, to minimize the radial temperature gradient, the denaturation zone has been further extended towards the center. Fig. (5) verifies the improvement brought about by the trenches in flattening out the temperatures in the distinct zones. The final design is investigated in Case 5, in which the number of heaters are increased to seven (refer to Tab. 1). Different heat flux values are assigned to the individual heaters and the values which result in a near-ideal temperature distribution in the microchannels are calculated. Fig. (6) depicts this near-ideal temperature distribution. The temperature profile within the three zones is almost flat and well within the required range and the transition time between the regions is also very short. As the results clearly show, having split heaters instead of a single heater for a given zone, facilitates application of different flux values and results in a perfect temperature distribution. This variable heating rate can be adjusted by a control circuit which would control the current flowing through each heating piece depending on the desired temperature within the zone. The temperature distribution on the surface of the device is also shown in Fig. (7). The split heaters and uniform temperature zones can be seen in the figure.

Since all the conclusions are based on the average temperature, the deviation of the actual temperature from the average for a given cross-section of the microchannel is examined at various locations. It is observed that the variation of temperature at a given cross-section is below $1{ }^{\circ} \mathrm{C}$. The temperature difference between the heater surface and the microchannel's bottom wall is also examined and a negligible temperature drop is observed. Therefore, in the real application measurement of the heater surface temperature would predict the temperature within the microchannel.

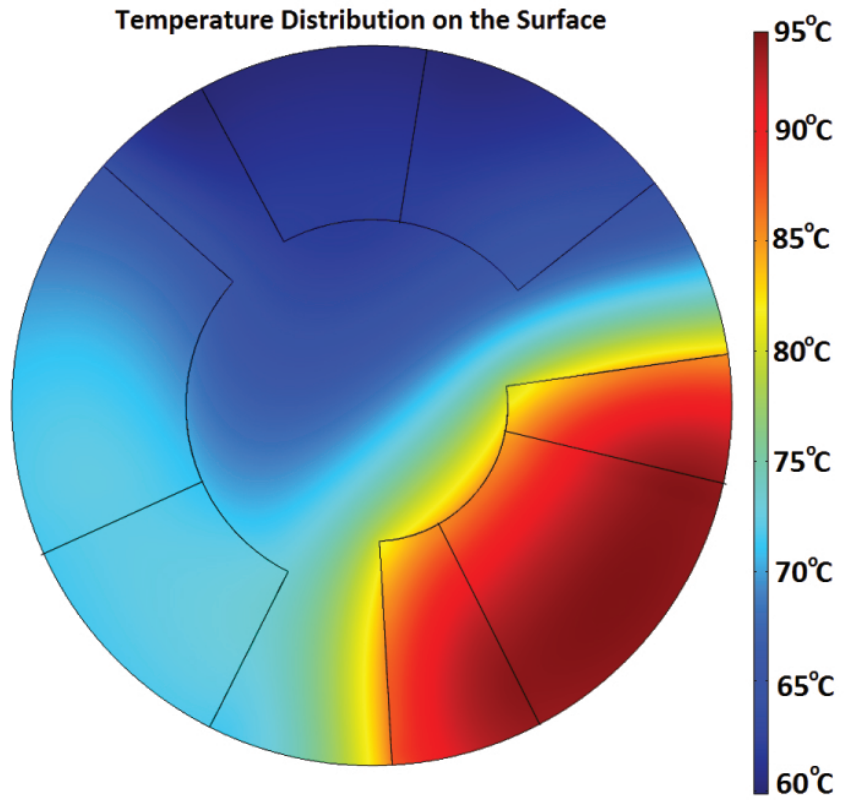

Figure 7. Temperature on the Surface of the Device

Based on results of these simulations, some further improvements can be implemented in the experimental phase of this project.

1. Before the beginning of the PCR cycling, an additional zone can be designated for an initial PCR activation $\left(95^{\circ} \mathrm{C}\right)$ for 5 minutes which would reduce the efforts for sample preparation. This initial activation step is implemented in the final design of the device and can be seen in Fig. 8.

2. The channel cross-sectional area can be increased gradually while moving towards the center of the spiral. This way, the residence time for each cycle can be kept constant which may improve the amplification efficiency.

3. Since this is a continuous flow device, the velocity profile is parabola-like laminar velocity profile. Although, our design depends on the average velocity, the residence time for DNA segments at different locations within the microchannel can be quite different. This issue can be resolved by switching to droplet based microfluidics. If the required enzyme molecules and targeted DNA can be trapped in a droplet, a droplet can travel through the heating zones. In this case the residence time of the DNA segments within a droplet would be equal which may again improve the amplification efficiency.

4. Driving the solution within a droplet can also facilitate multiplexing if different enzymes together with PCR solution can be trapped in different droplets in a controlled manner which would introduce a new dimension in microfluidic PCR technology. 


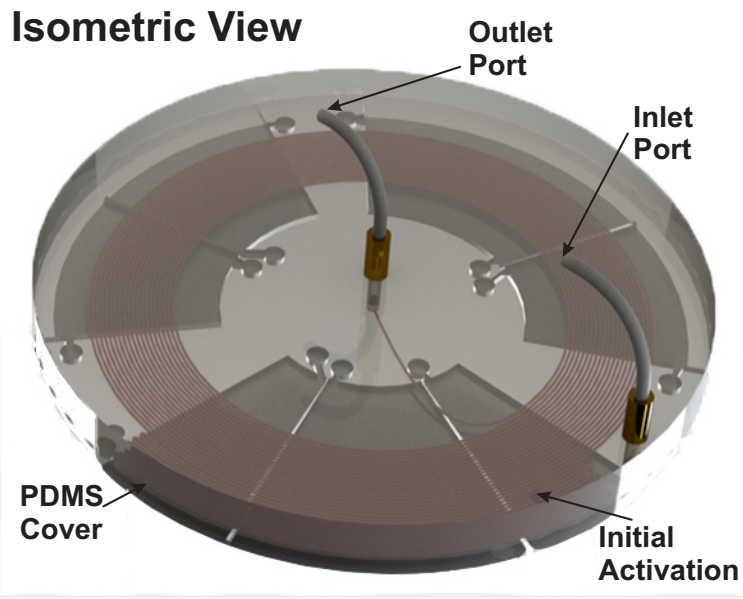

(a) Isometric view

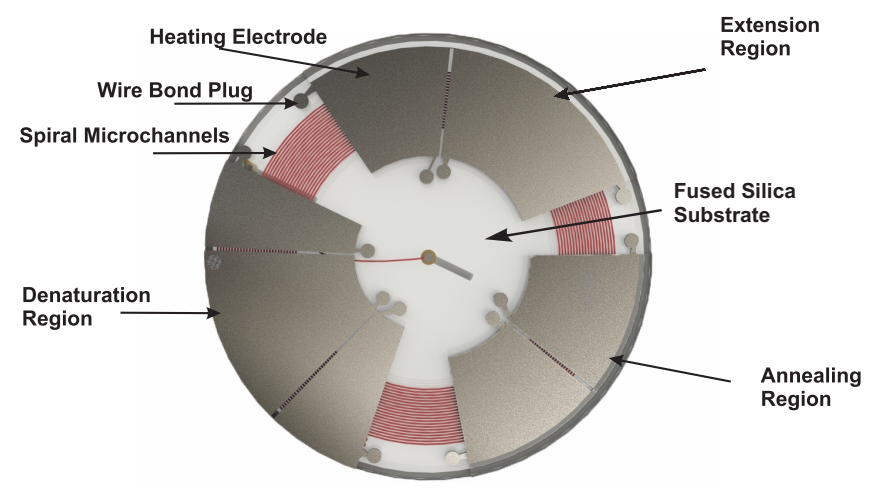

(b) Bottom view

Figure 8. CAD Drawing of the Proposed Device

\section{SUMMARY AND FUTURE WORKS}

In this study a microfluidics device with a spiral microchannel as the PCR reactor is analyzed. The flow and temperature fields are simulated within the device. The average temperature within the microchannel is determined, and by investigating the cyclic pattern of the average temperature some design parameters are optimized for possible enhanced performance. The device will be fabricated inside a glass substrate, as the low thermal conductivity of glass and good adhesion to the thin film heaters are important parameters in this design. Microchannels will be formed in the glass substrate by wet etching.The isolation trenches will also be wet-etched. Then, chromium thin films will be sputtered on the back-side of the substrate followed by etching to the desired configuration. A PDMS cover plate will be fabricated and bonded to the glass by plasma activation to form the channels. External tubing are to be connected to the channels via reservoir holes on the PDMS plate. Electrical connections will be achieved via wire bonding.

The temperature control of the distinct zones will be carried out by commercially available temperature control circuits. As the simulation predicts, the difference between the temperature of the heaters and the temperature of the fluid in the channel beneath the heater, is very small. As a practical consequence, the heater temperature can be measured and extrapolated to estimate the fluid temperature which is then fed to the control circuit. The second phase of the tests are to be carried out with actual biological samples, in which the amplification of the targeted DNA will be investigated.

\section{ACKNOWLEDGMENT}

The authors gratefully acknowledge financial support for Mr. Reza Salemmilani by Bilkent University Mechanical Eng. Department.

\section{REFERENCES}

[1] Weng, X., Jiang, H., and Li, D., 2011. "Microfluidic dna hybridization assays". Microfluidics and Nanofluidics, 11, pp. 367-383.

[2] Saiki, R. K., Scharf, S., Faloona, F., Mullis, K. B., Horn, G. T., Erlich, H. A., and Arnheim, N., 1985. "Enzymatic amplification of beta globin genomic sequences and restriction site analysis for diagnosis of sickle cell anemia". Science, 230, pp. 1350-1354.

[3] Udvardi, M. K., Czechowski, T., and Scheible, W.-R., July 2008. "Eleven golden rules of quantitative rt-pcr". The Plant Cell Online, 20(7), pp. 1736-1737.

[4] Hu, G., Xiang, Q., Fu, R., Xu, B., Venditti, R., and Li, D., 2006. "Electrokinetically controlled real-time polymerase chain reaction in microchannel using Joule heating effect". Analytica Chimica Acta, 557(1-2), pp. 146-151.

[5] Zhang, C., Xu, J., Wang, J., and Wang, H., 2007. "Continuous flow polymerase chain reaction microfluidics by using spiral capillary channel embedded on copper". Analytical Letters, 40(3), pp. 497-511.

[6] Zhang, C., and Xing, D., 2007. "Miniaturized pcr chips for nucleic acid amplification and analysis: latest advances and future trends". Nucleic Acids Research, 35(13), pp. 42234237.

[7] Xiaoyu, J., Zhiqiang, N., Wenyuan, C., and Weiping, Z., 2005. "Polydimethylsiloxane (pdms)-based spiral channel pcr chip". Electronics Letters, 41(16).

[8] Matsubara, Y., Kerman, K., Kobayashi, M., Yamamura, S., Morita, Y., and Tamiya, E., 2005. "Microchamber array based DNA quantification and specific sequence detection from a single copy via pcr in nanoliter volumes". Biosensors and Bioelectronics, 20(8), pp. 1482 - 1490. 
[9] Cady, N. C., Stelick, S., Kunnavakkam, M. V., and Batt, C. A., 2005. "Real-time PCR detection of listeria monocytogenes using an integrated microfluidics platform". Sensors and Actuators B: Chemical, 107(1), pp. $332-341$.

[10] Fraga, D., Meulia, T., and Fenster, S., 2008. Curr. Protoc. Essential. Lab. Tech. ch. Real-time PCR. 